\title{
Network Energy Consumption in Ad-hoc Networks Under Different Radio Models
}

\author{
Tao Yang $\nmid$, Leonard Barolli†, Makoto Ikeda $\nmid$, Arjan Durresi $\uparrow$, Fatos Xhafał‡ \\ $\dagger$ Graduate School of Engineering \\ Fukuoka Institute of Technology (FIT) \\ 3-30-1 Wajiro-Higashi, Higashi-Ku, Fukuoka 811-0295, Japan \\ E-mail: \{bd07003,bd07001\}@ws.ipc.fit.ac.jp \\ $\ddagger$ Department of Information and Communication Engineering \\ Fukuoka Institute of Technology (FIT) \\ 3-30-1 Wajiro-Higashi, Higashi-Ku, Fukuoka 811-0295, Japan \\ E-mail: barolli@ fit.ac.jp \\ $\dagger \dagger$ Department of Computer and Information Science \\ Indiana University Perdue University Indianapolis \\ 723 W. Michigan Street SL 280, Indianapolis, IN 46202, USA \\ Email: durresi@cs.iupui.edu \\ 施 Department of Languages and Informatics Systems \\ Polytechnic University of Catalonia \\ Jordi Girona 1-3, 08034 Barcelona, Spain \\ Email: fatos@1si.upc.edu
}

\begin{abstract}
In this work, we consider the behavior of a wireless adhoc network under TwoRayGround and Shadowing radio models. By means of simulations, we analyze the performance of three routing protocols: AODV, DSR, and DSDV, and study the global energy depletion inside the network. We found that the energy consumption due to AODV and $D S R$ can be very different with respect to the radio model. In particular, we found a maximum relative difference of $70 \%$. On the other hand, for the proactive DSDV routing protocol the energy consumption rate seems to be independent of the radio model, at least for moderate size of the network (256 nodes). Therefore, for small networks, although proactive protocols offer a great advantage in term of delay, the energy consumption is independent against link failures due to the irregularity of the radio medium.
\end{abstract}

\section{Introduction}

In recent years, technological advances have lead to the emergence of distributed Wireless Sensor and Actor Networks (WSANs) which are capable of observing the phys- ical world, processing the data, making decisions based on the observations and performing appropriate actions. These networks can be an integral part of systems such as battlefield surveillance and microclimate control in buildings, nuclear, biological and chemical attack detection, home automation and environmental monitoring $[1,2,3]$.

In WSANs, the phenomena of sensing and acting are performed by sensor and actor nodes, respectively. However, in some applications instead of actor nodes, integrated sensor/actor nodes which include both sensing and acting units can also be used. One of the examples of this kind of application is the distributed robot systems. In such systems, the robots which have both sensing and acting capabilities function as integrated sensor actor nodes.

Recently, we witnessed a lot of research effort towards the optimization of standard communication paradigms for WSN. In fact, the traditional Wireless Network (WN) design has never paid attention to constraints such as the limited or scarce energy of nodes and their computational power. Another aspect which is different from traditional WN is the communication reliability and congestion control. In traditional wired nets, in general the communication paths are stable along the transmission instances. This fact 
permits to use the end-to-end approach to the design of reliable transport and application protocols. The TCP works well because of the stability of links. On the other hand, in WSN and WSAN paths can change over time, because of time-varying characteristics of links and nodes reliability. These problems are important especially in a multi-hop scenario, where nodes accomplish also at the routing of other nodes' packets. Therefore, new routing and MAC protocols should be used to accommodate the requirements of ad-hoc WSN.

In this regard, we consider a WSAN, where actors performs both sensing and routing. The interconnection of actors can be considered as a standard ad-hoc network. In this paper, we concentrate in the sensing, routing and topology management of WSAN. So in the simulations, only the sensor-to-sink data transmission is considered. In the future work, we will consider also sensor-actor and actoractor communications.

The novelty of the paper is to study the energy consumption of ad-hoc networks under the assumption that the radio model presents some degree of irregularity, such as the Shadowing model. We perform the simulations also for TwoRayGround radio model and compare the performance of three protocols. As a technique for data transport, we use the packet repetition scheme. The repetition rate depends on a number of other factors, first of all the bound on the signal distortion perceived at the sink node. However, here we are interested in the depletion energy rate only.

The paper is organized as follows. In Sections 2, we explain the proposed network simulation model. In Section 3 , we present the event detection and transport. In Section 4, we discuss the simulation results. Conclusions of the paper are given in Section 5.

\section{Proposed Network Simulation Model}

For simplicity, we consider only the sensing part of the actor node. Every actor node performs both sensing and routing of data packets. Proposed network simulation model is shown in Fig. 1, while the sensor node model is shown in Fig. 2. In Fig. 2, the channel 1 is used for communication with neighbor nodes, while channel 2 for communication between present node and event node.

In our model, every node detects the physical phenomenon and sends back to the sink node data packets. We suppose that the sink node is more powerful than other nodes and it is always located a the borders of the service area. We analyze the performance of the network in a fixed time interval, $\tau$. This can be considered as the available time for the detection of the phenomenon and its value is application dependent.

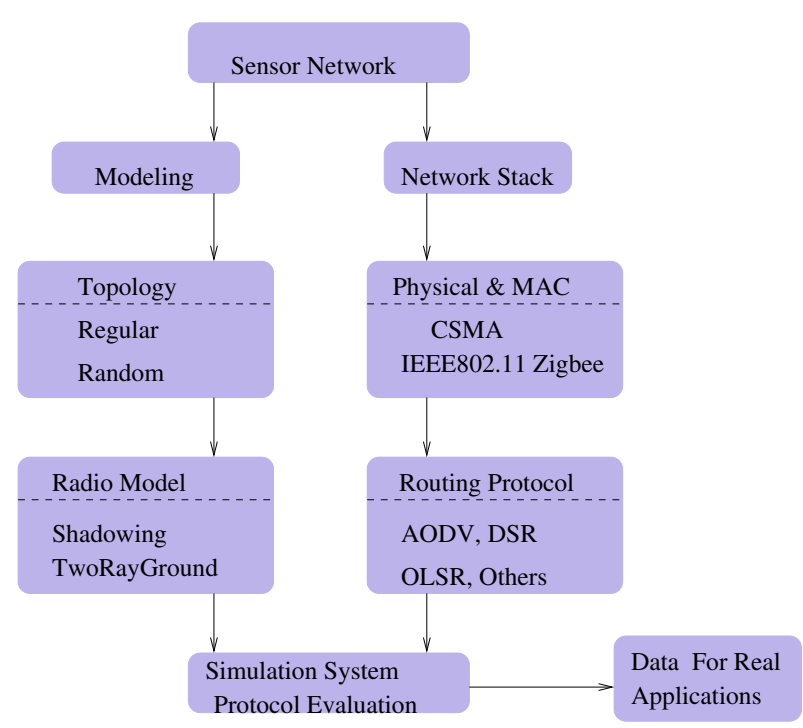

\section{Figure 1. Proposed network simulation} model.

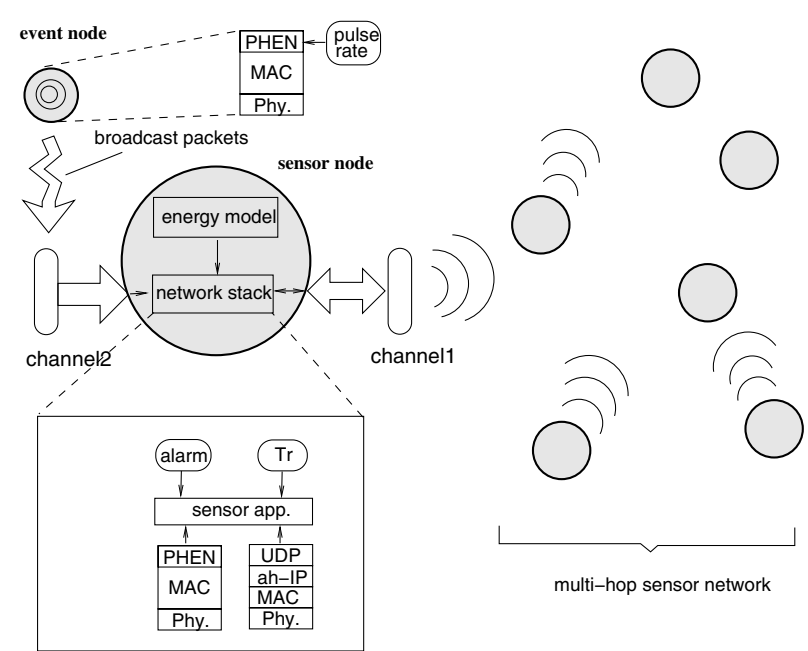

Figure 2. Sensor node model.

\subsection{Topology}

In this work, we consider a square grid topology. In this case, in order to guarantee the connectedness of the network we should set the transmission range of every node to the step size, $d$, which is the minimum distance between two rows (or columns) of the grid. In fact, by this way the number of links that every node can establish, a.k.a the node degree is $D=4$. By using Cooper's theorem [4] along with some power control techniques, one could use also $D=2^{1}$. However, we assume all nodes to be equal, therefore the degree is fixed to 4 . Nodes at the borders have $D=2$.

\footnotetext{
${ }^{1}$ By using the theorem in [4], we can say that a simple 2 regular network [5] is almost surely strongly 2 connected.
} 


\subsection{Routing Protocols}

We are aware of many routing protocols for ad-hoc networks during recent years. Here, we consider as reactive protocols Ad-hoc On-demand Distance Vector (AODV) and Dynamic Source Routing (DSR), while as proactive routing protocol the Destination Sequenced Distance Vector (DSDV).

AODV and DSR build up a route only when it is needed, i.e. when a node has data to send [6]. In AODV and DSR, there are two phases: Route Request (RReq) and Route maintenance. The RReq phase is accomplished by means of broadcast messages to neighbor nodes. In AODV, the destination node chooses one among all possible discovered routes. In DSR, the source node can learn multiple routes towards the destination. AODV maintains per destination routing tables, while DSR contains multiple routes cache entries for each destination. Moreover, in DSR there is not any mechanism to check whether a cached routed has become staled. For these on-demand protocols, it is expected that the impact of radio link dynamics on AODV is minimal, because of the multi-round mechanism. However, we show that the performances depend on the infrastructure too.

DSDV is a table-driven routing scheme. Each entry in the routing table contains a sequence number. The sequence numbers are generally even if a link is present, otherwise an odd number is used. The numbers are generated by the destination, and the emitter needs to send out the next update with this number. Routing information is distributed among nodes by sending advertisement messages infrequently and smaller incremental updates more frequently.

\subsection{Sensor Model and Phenomenon Model}

In order to simulate the detection of a natural event, we used the libraries from Naval Research Laboratory (NRL) [7]. In this framework, a phenomenon is modeled as a wireless mobile node. The phenomenon node broadcasts packets with a tunable synchrony or pulse rate, which represents the period of occurrence of a generic event ${ }^{2}$. These libraries provide the sensor model with an alarm variable. The alarm variable is a timer variable. It turns off the sensor if no event is sensed within an alarm interval. In addition to the sensing capabilities, every actor can establish a multi-hop communication towards the Monitoring Node (MN) by means of a particular routing protocol. This case is the opposite of the polling scheme. We used two kind of reactive protocols: AODV, DSR and one proactive protocol DSDV [8].

Although not optimal for multi-hops WSNs, we assume that the MAC protocol is the IEEE 802.11 standard. This

\footnotetext{
${ }^{2}$ As a consequence, this model is for discrete events. By setting a suitable value for the pulse rate, it is possible to simulate the continuous signal detection such as temperature or pressure.
}

serves to us as a baseline of comparison for other contention resolution protocols. The receiver node is supposed to receive correctly data bits if the received power exceeds the receiver threshold, $\gamma$. This threshold depends on the hardware $^{3}$. As reference, we select parameters values according to the features of a commercial device (MICA2 OEM). In particular, for this device, we found that for a carrier frequency of $f=916 \mathrm{MHz}$ and a data rate of $34 \mathrm{KBaud}$, we have a threshold (or receiver sensitivity) $\left.\gamma\right|_{d B}=-118 \mathrm{dBm}$ [9]. The calculation of the phenomenon range is not yet optimized and the phenomenon propagation is assumed to follow the propagation laws of the radio signals. In particular, the emitted power of the phenomenon is calculated according to a two-rays propagation model [10]. Here, we use the Shadowing model for the radio medium. In Shadowing model, the node received power is:

$$
\left.P_{r}(d)\right|_{\mathrm{dB}}=\underbrace{\left.P_{t}\right|_{\mathrm{dB}}-\beta_{0}-10 \alpha \log \left(\frac{d}{d_{0}}\right)}_{\text {deterministic part }}+\underbrace{S_{\mathrm{dB}}}_{\text {random part }}
$$

where $\beta_{0}$ is a constant. The term $S_{\mathrm{dB}}$ is a random variable, which accounts for random variations of the path loss. This variable is also known as log-normal Shadowing, because it is supposed to be Gaussian distributed with zero mean and variance $\sigma_{\mathrm{dB}}^{2}$, that is $S_{\mathrm{dB}} \sim \mathcal{N}\left(0, \sigma_{\mathrm{dB}}^{2}\right)$. Given two nodes, if $P_{r}>\gamma(\gamma$ is the hardware-dependent threshold $)$, the link can be established. The case of $\sigma=0, \alpha=4$, $d>d_{0}$ is also called the TwoRayGround model and it is a deterministic model.

Lattice Network $D=2$ guarantees a connected network. Thus, it suffices to guarantee a value of $\operatorname{Prob}(D \geq 2)$ as close as possible to 1 . Let us note that the link between any two nodes is a Bernoullian random variable with a certain probability $p$. If we consider only the closest neighbors, we have that $\operatorname{Prob}(D \geq 2) \geq \sum_{2 \leq k \leq 4}\left(\begin{array}{c}N \\ k\end{array}\right) p^{k}(1-p)^{N-k}$. For $p=0.95, \operatorname{Prob}(D \geq 2) \approx 0 . \overline{9} 9 \overline{9} 5$. Thus, based on the grid step $d$ and on Eq. (1), we can set the maximum transmission range by solving $p=\operatorname{Prob}\left\{\left.P_{r}(d)\right|_{\mathrm{dB}}>\left.\gamma\right|_{\mathrm{dB}}\right\}=$ 0.95. It is straightforward to show that:

$$
\begin{aligned}
\left.P_{t}(d)\right|_{\mathrm{dB}} & =\left[10 \alpha \log _{10} d+\left.\gamma\right|_{\mathrm{dB}}-\operatorname{erfc}^{-1}(2 p) \sqrt{(2)} \sigma\right] \\
& +\beta_{0}
\end{aligned}
$$

where $\operatorname{erfc}^{-1}$ is the inverse of the standard error function. Eq. (2) provides the transmission power of each node, given a transmission range and a probability or rate of coverage

\footnotetext{
${ }^{3}$ Other MAC factors affect the reception process, for example the Carrier Sensing Threshold (CST) and Capture Threshold (CP) of IEEE.802.11 used in Ns-2.
} 
Table 1. Topology settings.

\begin{tabular}{|c|c|}
\hline \multicolumn{2}{|c|}{ Lattice } \\
\hline \hline Step & $d=\frac{L}{\sqrt{N}-1} \mathrm{~m}$ \\
\hline Service Area Size & $L^{2}=(800 \times 800) \mathrm{m}^{2}$ \\
\hline Number of Nodes & $N=16,64,256$ \\
\hline Transmission Range & $r_{0}=d$ \\
\hline
\end{tabular}

Table 2. Radio model and system parameters.

\begin{tabular}{|c|c|}
\hline \multicolumn{2}{|c|}{ Radio Model Parameters } \\
\hline Path Loss Coefficient & $\alpha=2.7$ \\
\hline Variance & $\sigma_{\mathrm{dB}}^{2}=16 \mathrm{~dB}$ \\
\hline Carrier Frequency & $916 \mathrm{MHz}$ \\
\hline Antenna & Omni-directional \\
\hline Threshold (Sensitivity) & $\gamma=-118 \mathrm{~dB}$ \\
\hline \multicolumn{2}{|c|}{ Other Parameters } \\
\hline Reporting Frequency & $\overline{T_{r}=[0.1,1000] \mathrm{pps}^{1}}$ \\
\hline Interface Queue Size & 50 packets \\
\hline UDP Packet Size & 100 bytes \\
\hline Detection Interval $\tau$ & $30 \mathrm{~s}$ \\
\hline
\end{tabular}

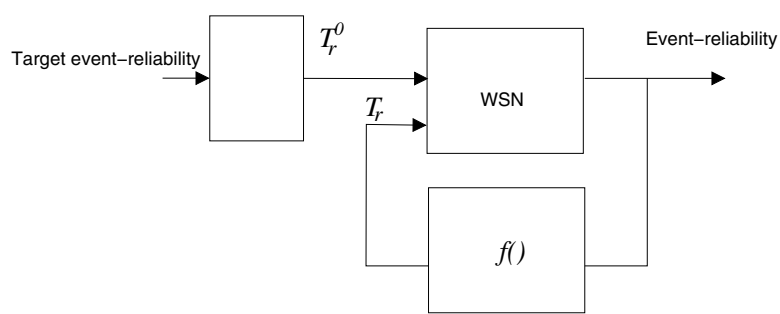

Figure 3. Data transport based on the eventreliability.

$p$. This should not be confused with the sensing coverage of ad-hoc network. An obvious effect of the Shadowing is the random coverage of the transmission range of each node. We will have different received powers in different directions. Consequently, the real coverage radius is not constant as in the ideal isotropic radiation case.

Energy Model A widely used energy model is as follows [11]. When a node transmits $k$ bits, the radio circuitry consumes an energy of $k P_{T x} T_{B}$, where $P_{T x}$ is the power required to transmit a bit which lasts $T_{B}$ seconds. By adding the radiated power $P_{t}(d)$, we have:

$$
E_{T x}(k, d)=k T_{B}\left(P_{T x}+P_{t}(d)\right) .
$$

Since packet reception consumes energy, by following the same reasoning, we have:

$$
\begin{aligned}
E(k, d) & =E_{T x}(k, d)+E_{R x}(k, d)=k P_{T x} T_{B}+k T_{B} P_{t}(d) \\
& +k P_{R x} T_{B}
\end{aligned}
$$

where $P_{R x}$ is the power required to correctly receive (demodulate and decode) one bit. In Tables 1 and 2, we present the values of parameters used in our model. Let us note that the power values concern the power required to transmit and receive one bit, respectively. They do not refer to the radiated power at all. This is also the energy model implemented in the widely used NS-2 simulator.

\section{Event Detection and Transport}

We use a data-centric model similar to [12], where the end-to-end reliability is transformed into a bounded signal distortion concept. In this model, after sensing an event, every node sends the data towards the MN. The transport used is a UDP-like transport, i.e. there is not any guarantee on the delivery of the data. While this approach reduces the complexity of the transport protocol and well fit the energy and computational constraints of nodes, the event-reliability can be guaranteed to some extent because of the spatial redundancy.

The node transmits data packets reporting the details of the detected event at a certain transmission rate ${ }^{4}$. The setting of this parameter, $T_{r}$, depends on several factors, as the quantization step of actors, the type of phenomenon, and the desired level of distortion perceived at the MN. In [13], the authors used this $T_{r}$ as a control parameter of the overall system. For example, if we refer to event-reliability as the minimum number of packets required at $\mathrm{MN}$ in order to reliably detect the event, then whenever the $\mathrm{MN}$ receives a number of packets less than the event-reliability, it can instruct sensor nodes to use a higher $T_{r}$. This instruction is piggy-backed in dedicated packets from the MN.

This system can be considered as a control system, as shown in Fig. 3, with the target event-reliability as input variable and the actual event-reliability as output parameter. The target event-reliability is transformed into an initial $T_{r}^{0}$. The control loop has the output event-reliability as input, and on the basis of a particular non-linear function $f(\cdot)$, $T_{r}$ is accordingly changed. We do not implement the entire control system, but only a simplified version of it. For instance, we vary $T_{r}$ and observe the behavior of the system in terms of the mean number of received packets. In other words, we open the control loop and analyze the forward chain only.

In this model, after sensing an event, every node establishes a UDP connection towards the sink node. The node

\footnotetext{
${ }^{4}$ Note that in the case of discrete event, this scheme is a simple packet repetition scheme.
} 
uses a repetition scheme for the packet transmission, i.e. it transmits multiple copies of the data packets reporting the details of the detected event [14]. On the other hand, the control of this parameter affects the congestion and the mean latency of transmissions towards the sink node. The dependence of the congestion on the irregularities of the radio medium is the core of our contribution and is explained in the simulation section.

\section{Simulations}

In this section, we present the simulation results of our proposed model. We simulated the network by means of Ns-2 simulator, with the support of NRL libraries ${ }^{5}$. As long as the network is being used, a certain amount of energy will be consumed. The energy consumption rate directly affects the life-time of the network, i.e. the time after which the network is unusable. The energy depletion is a function of the reporting rate as well as the density of the network nodes. Recall that the density of the network in the event-driven scenario correlates with the number of nodes that report their data. Accordingly, we define the energy consumed by the network in the detection interval $\tau$ as:

Consumed Energy $\triangleq$

$$
\frac{\text { Initial T. Energy - Final T. Energy }}{\tau},
$$

However, in order to compare the performance of the scaled networks, it is better to define the mean energy depletion rate per node as:

$$
\bar{\Delta}(\tau) \triangleq \frac{E_{I}-\bar{e}(\tau)}{\tau}=\frac{N E_{I}-\sum_{i=1}^{N} e_{i}(\tau)}{N \tau},
$$

where $e_{i}(\tau)$ is the node energy during detection interval $\tau$ and the means $(\bar{e}(\tau))$ are computed over the number of nodes. The $E_{I}$ is the initial energy of nodes. The number of nodes $N$ is set as power of integers in order to analyze the behavior of the scaled versions of the network. The initial position of the phenomenon node is varied along the simulation runs.

If all nodes have the same sensing range, the higher is the node density, the higher will be the number of nodes which can detect the phenomenon and the congestion level inside the network. There are analytical techniques to compute the minimum number of nodes to cover every point in a given area (see [16] and references therein), but all the available

\footnotetext{
${ }^{5}$ Since the number of scheduler events within a simulated network can be very high, we applied a patch against the scheduler module of NS-2 in order to speed up the simulation time [15]
}

results are for ideal radio model only. In regard of the energy depletion rate, we see from simulations that in the case of Shadowing model the network performs worse than in the deterministic path loss case.

In the deterministic case, the performance of the lattice depends on the lattice step and the path loss exponent. In fact, the energy loss can be formulated as follows. Given two configurations of the lattice with $d_{1}$ and $d_{2}$, respectively, it is straightforward to obtain the following relation between the consumed energies in a detection interval.

$$
\frac{E_{2}}{E_{1}} \sim\left(\frac{d_{2}}{d_{1}}\right)^{\alpha-2}
$$

For $\alpha>2$ and $d_{2}>d_{1}$, the energy loss is greater than 1 , therefore the small networks consume more than large ones ${ }^{6}$. Another explanation of this phenomenon is based on the theory of random graphs [17]. In this context, the network is modeled as a graph and the vertexes of the graph are nodes of the network. The parameter which affects the energy consumption is the hop count of the graph, that is the hop distance between two any nodes. In a pure random graph, where links are uncorrelated, the mean hop count is $\bar{h} \sim \frac{\ln N}{\ln D}$, while in a lattice with correlated links and $D=4$ as in our case, the mean hop count is $\bar{h} \sim O(\sqrt{N})$, which is greater than that of a pure random graph. However, in the case of Shadowing, the behavior of the graph tends to that of a random graph and then $\bar{h}$ decreases. The simulations reveal that for large scale networks the relative difference between deterministic and Shadowing model can be as high as $50 \%$.

Lattice Network For each routing protocol, the sample averages of Eq. (5) are computed over 20 simulation runs, and they are plotted in Fig. 4 to Fig. 9, with respect to the particular radio model used. We also show the $95 \%$ confidence interval of the sample averages. We can clearly distinguish three operating zones. For low values of $T_{r}$, the network is uncongested. At a particular value of $T_{r}$ ( $\sim 1 \mathrm{pps})$, the network reaches the maximum capacity. For $T_{r}>10 \mathrm{pps}$, contention and congestion periods augment, and consequently we need more energy in order to resolve packet loss by means of retransmissions. Although these three zones are present regardless of the radio model, we used two radio models: TwoRayGround and Shadowing.

In case of TwoRayGround, the energy consumption rate increases with N, as shown in Fig. 4, Fig. 5 and Fig. 6. For small number of nodes DSR has better behavior than the other protocols. However, when the number of nodes is 256 , DSDV consumes less energy than the other protocols. In the case of Shadowing as shown in Fig. 7, Fig. 8 and Fig. 9, the

\footnotetext{
${ }^{6}$ This is the worst case, because we supposed a global flooding in a time unit.
} 
consumed energy of AODV is less than the other protocols. While, the DSDV protocol consumes more energy than the other protocols.

The explanation of this effect is not simple, because it is intermingled with the dynamics of MAC and routing protocols. However, intuitively we can say that in the case of Shadowing the on-demand routing protocols are affected by the presence of Shadowing-induced unidirectional links. It is worth noting that AODV and DSR cannot use unidirectional links. On the other hand, exploiting such links is possible but the performance gains are quite low, because the routing protocol spends most of the time in the searching of a bi-directional path. Thus, given a fixed detection interval, $N_{r}$ can be much lower than its value in the case of ideal radio model, i.e. the TwoRayGround model, where the discovered paths do not change over time ${ }^{7}$. This fact may not affect the performance of the network, because it depends on the requirements of the application. For high values of $N$, the augmented interference level and the path instability seem to be predominant [18].

In NS-2, the Shadowing is considered time-varying, i.e. at every packet transmission the path loss is re-computed. Hence, the coverage is also time-varying. Although a timevarying Shadowing might appear unrealistic, the same result arises if we consider other more abstract models such as the Cerpa's model [19]. The occurrence of this phenomenon increases with $T_{r}$. However, after $N=16$, the number of collisions decreases, either because every node is silenced by an increased number of neighboring nodes, due to the Shadowing, and because fewer UDP packets are sent. Consequently, in the former case, nodes will defer the transmission of packets more frequently, by inducing less collisions as overall effect. Since the maximum number of collisions is still higher than that in the case of TwoRayGround model, the delay is increased remarkably as shown in Fig. 10. Note that in the case of $N=256$, we did not show any data, because the number of samples is not enough to draw any statistical evaluation. In this case, the values of the eventto-sink delay span the entire $[0,10] \mathrm{s}$ range. This means that most of time, when the MN receives packets, most of the delays are high.

\section{Conclusions}

In this paper, we considered the behavior of three adhoc network protocols under different radio models such as TwoRayGround and Shadowing. By using NS-2 simulator, we analyzed the performance of AODV, DSR, and DSDV protocols, and studied the global energy depletion inside the network. To the best knowledge of the authors, only the works in $[20,21]$ addressed the same problem, but in these

\footnotetext{
${ }^{7}$ This is true if we do not count the reliability of nodes, i.e. the probability of node failure.
}

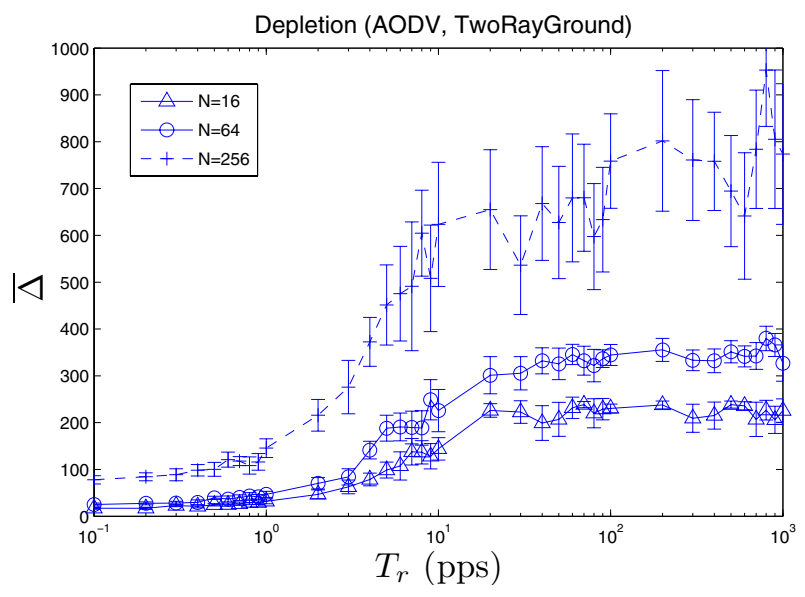

Figure 4. Sample averages of depletion for AODV (TwoRayGround).

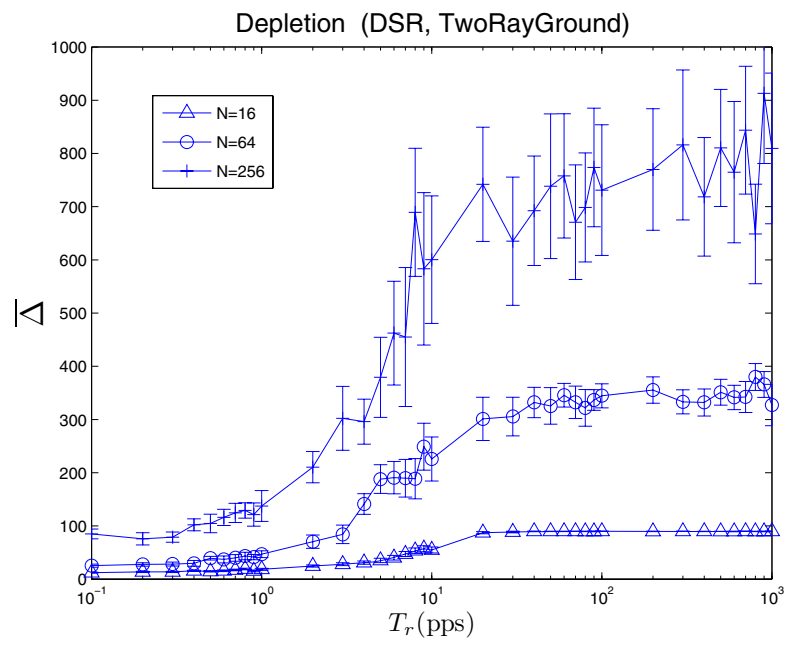

Figure 5. Sample averages of depletion for DSR (TwoRayGround).

works the effects of the irregularities of radio model have not been considered.

From the simulation results, we concluded as follows.

- The consumed energy rate depends on the radio irregularities for AODV and DSR, while for DSDV the consumed energy seems to be insensitive of the radio model.

- The energy consumption due to AODV and DSR can be very different with respect to the radio model. In particular, we found a maximum relative difference of $70 \%$. 


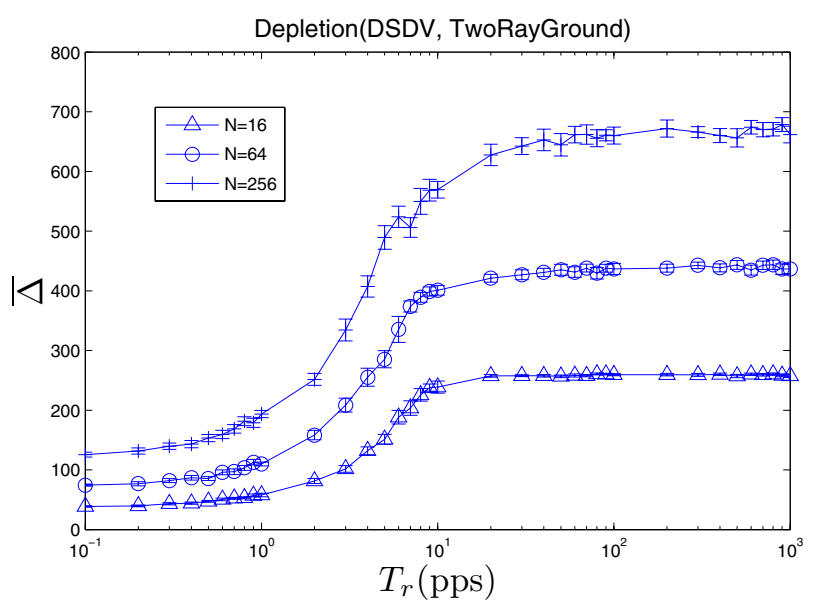

Figure 6. Sample averages of depletion for DSDV (TwoRayGround).

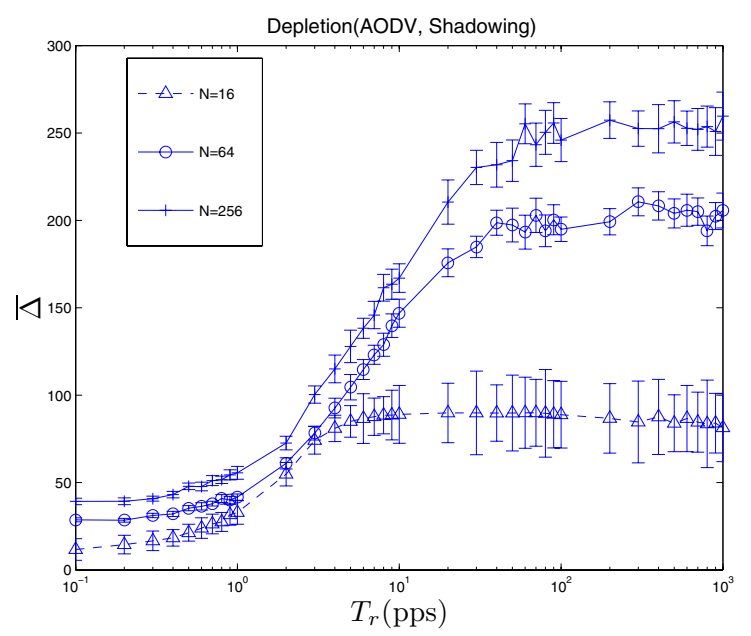

Figure 7. Sample averages of depletion for AODV (Shadowing).

- For the proactive DSDV routing protocol, the energy consumption rate seems to be independent of the radio model, at least for moderate network size. Therefore, for small networks, although proactive protocols offer a great advantage in term of delay, the energy consumption is independent against link failures due to the irregularity of the radio medium.

- For TwoRayGround model, the energy consumption rate increases with $\mathrm{N}$. For small number of nodes DSR has better behavior than the other protocols. However, when the number of nodes is 256, DSDV consumes less energy than the other protocols.

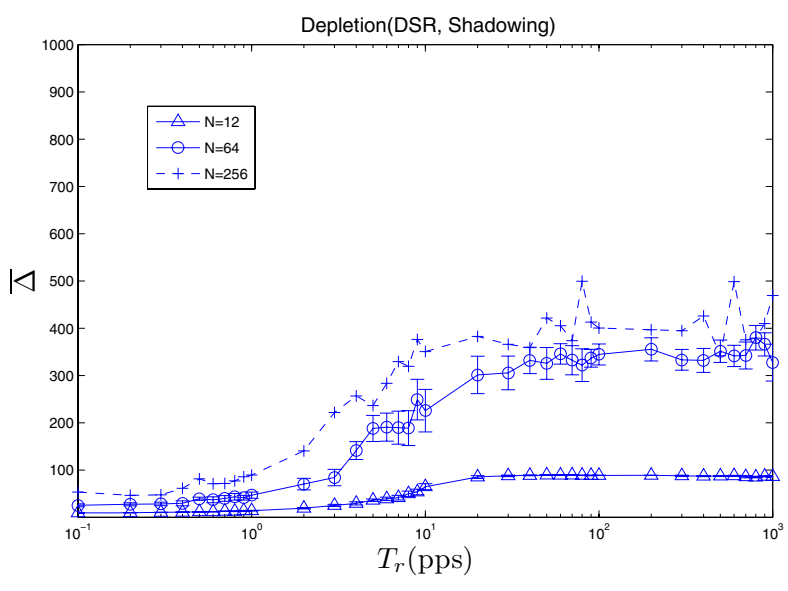

Figure 8. Sample averages of depletion for DSR (Shadowing).

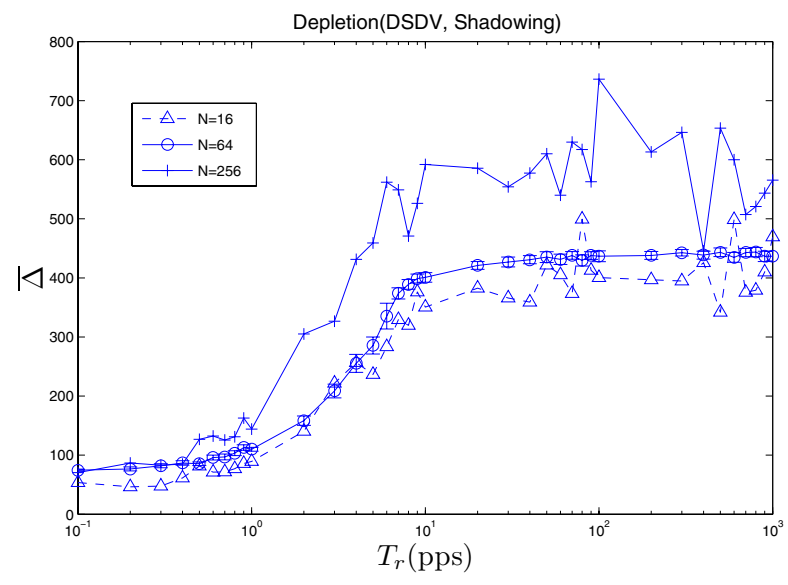

Figure 9. Sample averages of depletion for DSDV (Shadowing).

- For Shadowing model, AODV consumes less energy than the other protocols. While, DSDV protocol consumes more energy than the other protocols.

- For Shadowing model, the on-demand routing protocols are affected by the presence of Shadowinginduced unidirectional links. It is worth noting that AODV and DSR cannot use unidirectional links.

In our future work, we are planning to extend the results to other routing and MAC protocols and other non-lattice topologies. 


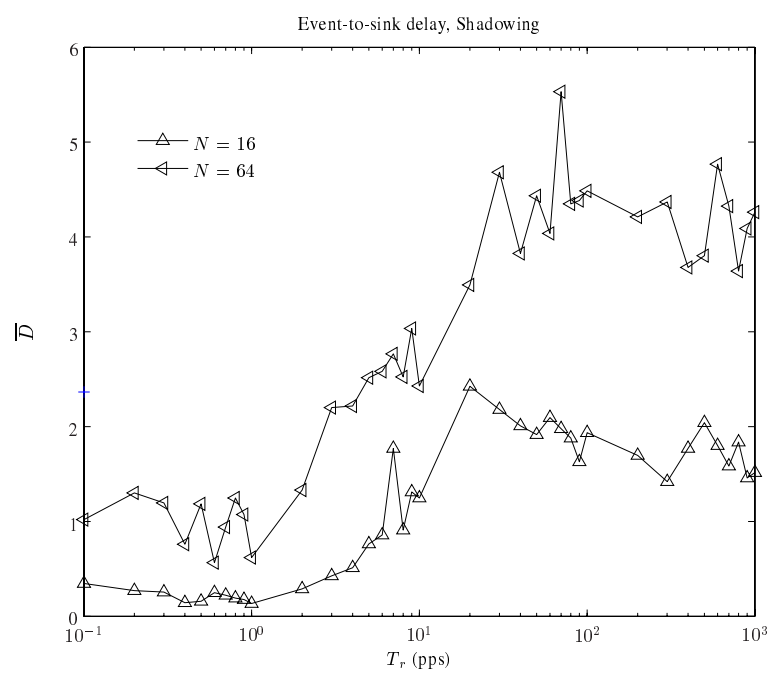

Figure 10. Average delay for AODV.

\section{Acknowledgment}

This work is partially supported by International Communications Foundation (ICF) of Japan and Japanese Society for the Promotion of Science (JSPS). The authors would like to thank ICF and JSPS for the financial support.

\section{References}

[1] I. F. Akyildiz, I. H. Kasimoglu, "Wireless Sensor and Actor Networks: Research Challenges", Ad Hoc Networks Journal (Elsevier), Vol. 2, No. 4, pp. 351-367, October 2004.

[2] O. Younis, S. Fahmy, "HEED: A Hybrid, Energy-efficient, Distributed Clustering Approach for Ad-hoc Sensor Networks", IEEE Transactions on Mobile Computing, Vol. 3, No. 4, pp. 366-379, 2004.

[3] G. W.-Allen, K. Lorincz, O. Marcillo, J. Johnson, and M. Ruiz an J. Lees, "Deploying a Wireless Sensor Network on an Active Volcano", IEEE Internet Computing, Vol. 10, No. 2, pp. 18-25, March 2006.

[4] C. Cooper, "A Note on the Connectivity of 2-Regular Digraphs", Random Structures Algorithms, Vol. 4, pp. 469472, 1993.

[5] T. Yang, G. De Marco, M. Ikeda, and L. Barolli, "A Case Study of Event Detection in Lattice Wireless Sensor Network with Shadowing-induced Radio Irregularities", Proc. of International Conference on Advances in Mobile Computing and Multimedia (MoMM-2006), pp. 241-250, December 2006

[6] C. Perkins, Editor, "Ad Hoc Networks", Addison-Wesley, 2001.

[7] I. Donward, NRL's Sensor Network Extension to NS-2, Available on line at http://pf.itd.nrl.navy.mil/nrlsensorsim/, 2004.
[8] DSDV, Available on line at http://decision.csl.uiuc.edu/ wireless/dsdv/.

[9] Crossbow Technology, Inc., Available on line at http://www. xbow.com/.

[10] T.S. Rappaport, "Wireless Communications", Prentice Hall PTR, 2001.

[11] W. Ye, J. Heidemann, and D. Estrin, "Medium Access Control with Coordinated Adaptive Sleeping for Wireless Sensor Networks", IEEE/ACM Transaction Networking, Vol. 12, No. 3, pp. 493-506, 2004.

[12] W. B. Heinzelman and A. P. Chandrakasan and H. Balakrishnan, "An Application-specific Protocol Architecture for Wireless Microsensor Networks", IEEE Transactions on Wireless Communications, Vol. 1, No. 4, pp. 660-670, October 2004.

[13] Ö. B. Akan and I. F. Akyildiz, "Event-to-sink Reliable Transport in Wireless Sensor Networks", IEEE/ACM Transactions on Networking, Vol. 13, No. 5, pp. 1003-1016, 2005.

[14] M. C. Vuran, V. C. Gungor, and O. B. Akan, "On the Interdependency of Congestion and Contention in Wireless Sensor Networks", Proc. of CST SenMetrics-2005, Available on line at http://users.ece.gatech.edu/mcvuran/InterdepSenMetrics.pdf, July 2005.

[15] D. X. Wei, "Speeding up Ns-2 Scheduler", Avaiable on line at http://netlab.caltech.edu/weixl/technical/ns2patch, September 2005.

[16] H. Zhang and J. C. Hou, 'Is Deterministic Deployment Worse than Random Deployment for Wireless Sensor Networks?", Proc. of IEEE INFOCOM-06, pp. 1-13, April 2006.

[17] B. Bollobas, "Random Graph", Academic Press,1985.

[18] T. Yang, G. De Marco, M. Ikeda, and L. Barolli, Impact of Radio Randomness on Performances of Lattice Wireless Sensors Networks based on Event-Reliability Concept, International Journal of Mobile Information Systems (IJMIS), Vol. 2, No. 4, pp. 211-227, October-December 2006.

[19] A. Cerpa, J. L. Wong, L. Kuang, M. Potkonjak, D. Estrin, "Statistical Model of Lossy Links in Wireless Sensor Networks", Proc. of Fourth International Symposium on Information Processing in Sensor Networks (IPSN-2005), pp. 8188, 2005.

[20] G. Zhou, T. He, S. Krishnamurthy, J. A. Stankovic, "Models and Solutions for Radio Irregularity in Wireless Sensor Networks", ACM Transaction on Sensors Network, Vol. 2, No. 2, pp. 221-262, 2006.

[21] M. Zuniga and B. Krishnamachari, "An Analysis of Unreliability and Asymmetry in Low-power Wireless Links", Available on line at http://www-scf.usc.edu/marcozun/, 2006. 